\title{
Serum Levels of the Biomarkers Associated with Astrocytosis, Neurodegeneration, and Demyelination: Neurological Benefits of Citicoline Treatment of Patients with Ischemic Stroke and Atrial Fibrillation
}

\author{
O. V. Kuryata, ${ }^{1}$ Yu. S. Kushnir, ${ }^{1}$ V. S. Nedzvetsky, ${ }^{2,3}$ \\ V. V. Korsa, ${ }^{4}$ and A. A. Tykhomyrov ${ }^{4}$
}

Received October 19, 2020

Ischemic stroke is a main complication of atrial fibrillation (cardiac arrhythmia). The aim of our study was to estimate the effects of citicoline (CDP-choline) therapy on the levels of circulating neurospecific protein markers in serum of the patients with ischemic stroke and atrial fibrillation. Fiftyfour patients (mean age 76 years) treated with citicoline in a dose of $2.0 \mathrm{~g}$ daily intravenously for 12 to 14 days in addition to basic treatment formed the examined group. Thirty-two patients (mean age 68.5 years) obtained only standard therapy and formed the control group. Serum levels of neuronal and glial protein markers, including glial fibrillary acidic protein (GFAP), a neurofilament light subunit (NF-L), myelin basic protein (MBP), and ionized calcium-binding adaptor molecule 1 (Iba1), were measured in patients of both groups before and after treatment; an immunoblotting technique followed by densitometry analysis were used. Supplementary citicoline treatment provided significant reductions of the levels of GFAP $(33 \%, P=0.034)$, NF-L $(27 \%, P=0.019)$, and MBP $(32 \%, P=0.018)$, as compared to the initial values, while there were no marked changes in the studied parameters in the control group. The results obtained allow us to hypothesize that therapeutic benefit of citicoline in patients with ischemic stroke and atrial fibrillation can be mediated through increasing neuronal viability, protecting against axonal injury, decreasing the level of reactive astrogliosis, preventing deficiencies in the blood-brain integrity, and reducing the intensity of demyelination. However, citicoline administration exerted no effect on the blood content of microglial marker Iba-1, thus possibly preserving an important functional significance of microglia, which is needed to resolve local inflammation and clear cellular debris, and also provide protective factors to reduce cell injury in the ischemic brain. The obtained results indicate that serum levels of neurospecific biomarkers are significant and clinically relevant indices of the efficiency of treatment of the above-mentioned pathologies and can be used for further investigations of the stroke pathophysiology and molecular mechanisms of nootropic-mediated neuroprotection.

Keywords: ischemic stroke, atrial fibrillation, citicoline, glial fibrillary acidic protein (GFAP), neurofilaments (NFs), myelin basic protein (MBP), ionized calcium-binding adaptor molecule 1 (Iba1)

\section{INTRODUCTION}

Atrial fibrillation (AF) is the most common clinically significant cardiac arrhythmia, which is estimated to affect 33 million people worldwide and considered as a "cardiovascular epidemy" of the XXI

\footnotetext{
${ }^{1}$ Dnipro State Medical University, Dnipro, Ukraine.

${ }^{2}$ Bingöl University, Bingöl, Turkey.

${ }^{3}$ Oles Honchar Dnipro National University, Dnipro, Ukraine.

${ }^{4}$ Palladin Institute of Biochemistry, National Academy of Sciences of Ukraine, Kyiv, Ukraine.

Correspondence should be addressed to A.A. Tykhomyrov

(e-mail: artem_tykhomyrov@ukr.net).
}

century [1]. This pathology is a well-established risk factor for other cardiovascular diseases, including heart failure and thromboembolic stroke or systemic thromboembolism, especially in the elderly. It has been estimated that AF accounts for approximately one-third of all stroke cases in 80- to 89-year-old individuals [2, 3]. Meanwhile, stroke is the third leading cause of death after ischemic heart disease and cancer; it leads to permanent disabilities in $80 \%$ of the survivors [4]. A few studies demonstrated that, apart from neurons, other cells are involved in the pathogenesis of ischemia, including the effects of a detrimental interaction between glia, neurons, vascular cells, and matrix components, which is 
collectively referred to as a "neurovascular unit" [5].

Thus, AF is the most important risk factor associated with acute stroke; however, the severity of stroke $+\mathrm{AF}$ is generally greater than that of strokes of other types. Growing evidence indicates that AF can exert adverse effects beyond an increased risk of cardiovascular diseases [6, 7]. Several studies have found a significant association between AF and total brain atrophy [8, 9]. Moreover, data have emerged showing that AF may be a risk factor for cognitive impairment and strokeindependent dementia, indicating that arrhythmia exerts considerable additional detrimental effects on the brain [10]. Stroke and subclinical infarcts related to a hypercoagulation state, circulatory stasis, and endothelial injury may explain the association of AF with multi-infarct dementia $[3,8]$.

Protection of the above-mentioned complex (neurovascular unit) is, therefore, a potentially effective strategy to preserve a delicate balance of the blood supply and energy requirements in the microenvironment of the brain, thereby providing limitation of the neurological deficits and neuron death after an ischemic insult. Systemic anticoagulation therapy provides effective reduction of the risk of stroke in AF patients. Such therapy, however, has several drawbacks related to negative side effects of direct oral anticoagulants $[2,11]$. It can be believed that endogenous regeneration and neuronal functional improvement can be achieved by some agents clinically belonging to other therapeutic categories. For example, one of the new nootropic drugs, which might combine neurovascular protection and a repair effect, is cytidine-5' -diphosphate choline (CDP-choline), or citicoline [12]. This agent first identified in 1955 by Kennedy and colleagues as a key intermediary in the biosynthesis of phosphatidylcholine, was synthesized in 1956 and has been intensely studied in Europe, Japan, and USA for several decades [13]. As was found, citicoline provided continuous neuroprotective benefit in different clinical stroke trials [14].

Investigation of the molecular mechanisms contributing to neural cell damage and/or death under stroke conditions is an important issue in formulating effective neuroprotective strategies and regimes. Due to ischemic cell injury and disruption of the blood-brain barrier (BBB), some specific proteins are released from neurons and glial cells into the bloodstream, so that they can be utilized as plausible markers for the diagnosis and prognosis in patients with ischemic stroke $[15,16]$. Among all neuronal proteins, those of neurofilaments (NFs) are promising candidates on the role of biomarkers of axonal injury and neuronal loss because they are expressed exclusively in neurons $[17,18]$.

Numerous studies indicated that glial fibrillary acidic protein (GFAP), a specific protein of astroglial cells, can serve as a sensitive and valuable marker of reactive astrocytosis and $\mathrm{BBB}$ damage [19]. An injury of the white matter due to oligodendrocyte death is an integral component of most strokes in humans, which contributes significantly to long-term sensorimotor and cognitive deficits [20].

Elevated serum levels of myelin basic protein (MBP) were usually observed in traumatic brain injury, benign and malignant intracranial tumours, CNS infections, and cerebrovascular accidents; the respective index is used as a specific marker of demyelination [21].

Microglial cells are resident immune cells within the CNS, which play a dual role in the initiation and propagation of inflammatory responses in ischemic lesions [22]. Activated microglia release reactive oxygen species (ROS) and pro-inflammatory cytokines, which are harmful for surrounding neurons, astrocytes, and oligodendrocytes. On the other hand, microglia may counteract local inflammation, clear cellular debris, and release protective factors capable of reducing the reactivity of astrocytes and diminishing oligodendrocyte injury and/or demyelination [23].

Ionized calcium-binding adaptor molecule 1 (Iba1) is a microglia/macrophage-specific calciumbinding protein, which is necessary for phagocytosis of activated microglia [24]. Both GFAP and Iba1 have been recently demonstrated as additional biomarkers related to neuroinflammation after mild traumatic brain injury [25].

Thus, in order to evaluate the efficacy of citicoline treatment in post-stroke patients with AF and to explore molecular mechanisms of probable benefits of this agent, serum levels of clinically relevant biomarkers (GFAP, NF-L, MBP, and Iba-1) were estimated in the respective patient group.

\section{METHODS}

Ninety-six patients (44 men and 52 women, 64 to 78 years old) suffering from ischemic stroke 
combined with AF were admitted to the hospital with in an acute phase; these patients formed the studied group. The presence of AF was established in accordance with recommendations of the European Society of Cardiology [26, 27]. Acute ischemic stroke was diagnosed by a neurologist basing on the clinical symptoms and data of computer tomography. All patients had permanent and persistent forms of AF.

All patients admitted to the hospital with acute ischemic stroke were randomly divided into two groups. Group $1(n=54)$ was formed from the patients subjected to standard acute stroke treatment, who received additionally citicoline as a drug solution "Neurocytin ${ }^{\circledR}$ " produced by Yuria-Farm Ltd. (Ukraine). The patients received citicoline (2.0 g daily) via slow i.v. infusions of $100 \mathrm{ml} 1 \%$ solution twice a day. The duration of such treatment was 12 to 14 days. Standard treatment of acute ischemic stroke included introductions of antihypertensive drugs and anticoagulants and also antiplatelet therapy in accordance with the Guidelines for the Early Management of Acute Ischemic Stroke [28]. Patients of group 2 obtained only the above-described standard treatment. The corresponding demographic and clinical parameters of both groups are presented in Table 1. As can be seen, the two groups demonstrated no significant intergroup differences from any aspect.

After overnight fasting, initial blood samples of each subject were taken from their antecubital vein and collected into BD Vacutainer venous blood collection tubes containing a clot activator. Blood samples from the control and citicoline-treated patients were collected after an overnight fasting the day after last injection of citicoline. Blood was allowed to form a clot at room temperature for $60 \mathrm{~min}$, and then the samples were centrifuged at
$3,000 \mathrm{~g}$ for $10 \mathrm{~min}$ at $15^{\circ} \mathrm{C}$ to separate the serum. Analysis of the routine biochemical parameters was performed by a standard analytical technique without freezing, while $1.0 \mathrm{ml}$ of serum samples was aliquoted and immediately frozen at $-20^{\circ} \mathrm{C}$ until immunochemical examination of the biomarkers. The concentration of total serum protein was measured by the spectrophotometrical Stoscheck method [29].

Serum samples were diluted 1:5 with SDScontaining reducing electrophoretic Laemmli sample buffer (160 mM Tris-HCl, pH 6.8, 4\% SDS, $10 \% \beta$-mercaptoethanol, and $20 \%$ glycerol) and boiled for $5 \mathrm{~min}$. Equal amounts of the proteins (100 $\mu \mathrm{g} /$ track) were loaded onto $10 \%$ SDSPAGE and electrophoresed. After electrophoresis, proteins were transferred onto nitrocellulose membranes with the pore diameter of $0.45 \mu \mathrm{m}$ (GE Healthcare, Amersham Bioscience, Great Britain) by electroblot. Then, the membranes were blocked in $5 \%$ solution of fat-free skim milk in phosphate buffered saline containing $0.05 \%$ Triton $\mathrm{X}-100$ (PBST) at $37^{\circ} \mathrm{C}$ for $90 \mathrm{~min}$. After blocking, the membranes were incubated with primary antibodies to proteins of interest, namely anti-GFAP (Santa Cruz Biotechnology, USA, cat. No. sc-9065), anti-NF-L (Santa Cruz Biotechnology, USA, cat. No. sc-20012), anti-MBP (Invitrogen, USA, cat. No. PA5-79642), or anti-Iba-1 (Invitrogen, USA, cat. No. MA5-27726) overnight at $4{ }^{\circ} \mathrm{C}$. After washing in PBST, the membranes were incubated with the appropriate anti-rabbit or anti-mouse secondary antibodies at $37^{\circ} \mathrm{C}$ for $90 \mathrm{~min}$ and subjected to several washes in PBST. Finally, specific immunostaining was developed by enhanced chemiluminescence detection by incubation with the HPR substrate $(0.1 \mathrm{M}$ Tris- $\mathrm{HCl}, \mathrm{pH} 8.5,0.25 \mathrm{M}$ luminol, $0.09 \mathrm{M}$ coumaric acid, and $0.0035 \% \mathrm{H}_{2} \mathrm{O}_{2}$ )

T a b 1 e 1. Demographic and Clinical Characteristics of Two Groups of the Patients with Acute Ischemic Stroke Combined with Atrial Fibrillation

\begin{tabular}{lccc|c}
\hline Parameter & Total & Group 1 $(n=54)$ & Group 2 $(n=42)$ & $P$ \\
\hline Males & $44(45.4 \%)$ & $24(44.4 \%)$ & $20(47.6 \%)$ & 0.76 \\
Females & $52(54.6 \%)$ & $30(55.6 \%)$ & $22(52.4 \%)$ & 0.14 \\
Age & $75.0[65.0 ; 78.0]$ & $76.0[67.0 ; 78.0]$ & $68.5[64.2 ; 78.0]$ & 0.95 \\
Body Mass Index & $27.8[24.9 ; 31.1]$ & $27.9[24.8 ; 31.0]$ & $27.7[24.9 ; 31.4]$ & - \\
Atrial Fibrillation & $96(100.0 \%)$ & $54(100.0 \%)$ & $42(100.0 \%)$ & - \\
Arterial Hypertension & $96(100.0 \%)$ & $54(100.0 \%)$ & $42(100.0 \%)$ & - \\
Coronary Artery Disease & $96(100.0 \%)$ & $54(100.0 \%)$ & $42(100.0 \%)$ & 0.96 \\
Heart Failure & $80(82.5 \%)$ & $39(72.2 \%)$ & $40(95.2 \%)$ & 0.09 \\
Diabetes mellitus & $29(29.9 \%)$ & $12(22.2 \%)$ & $16(38.1 \%)$ & \\
\hline
\end{tabular}

Footnote: Limites of quartiles for distributions of age and BMI are shown in brackets. 
followed by exposure on X-ray films (Konica Minolta, Japan). Determination of the molecular masses of the target proteins was performed by comparison with migration of standard markers (10-270 kDa, PageRuler Prestained Protein Ladder, Fermentas, Lithuania). Protein bands were digitized and quantified with the use of TotalLab software (version 120, TotalLab Ltd., USA). The results were expressed as arbitrary units of the optical density.

Relative amounts of the neurospecific protein markers were graphically presented as 'box-withwhiskers' plots, which display mean values, medians, standard deviations (s.d.), and standard errors of the mean (s.e.m.). The paired Student's $t$-test was used to evaluate the significance of differences between studied values for each patient before and after treatment. All calculations were performed using OriginPro software (version 8.6). Intergroup differences with $P<0.05$ were considered statistically significant.

\section{RESULTS}

Images of the representative blotorgams of circulating neurospecific proteins accompanied by results of densitometric analysis are shown in Figs. 1-4. The serum GFAP immunoreactivity was recognized as that related to a single polypeptide with $\mathrm{M}_{\mathrm{m}}=49 \mathrm{kDa}$ (Fig. 1). The citicoline treatment provided a significant decrease ( on average, by $34 \%$ ) in the average GFAP level in the patients' serum, from the initial value $0.86 \pm 0.03$ to $0.57 \pm 0.09$ a.u. after treatment $(P=0.034)$.

The NF-L polypeptide was found in the serum as a band with $M_{m}$ of $68 \mathrm{kDa}$ (Fig. 2). Moderate but statistically significant down-regulation of the level of this neurofilament protein was observed after citicoline treatment (a $27 \%$ decrement, from $0.63 \pm 0.06$ to $0.46 \pm 0.06$ a.u., $P=0.019$ ).

The immunoblot analysis revealed high amounts of MBP polypeptides with $\mathrm{M}_{\mathrm{m}} 125$ and $90 \mathrm{kDa}$ in the patients' serum (Fig. 3). Citicoline supplementation markedly reduced the mean total level of MBP polypeptides (a $35 \%$ decrease, from $0.26 \pm 0.03$, on average, to $0.17 \pm 0.02$ a.u., $P=0.018$ ).

Iba-1 polypeptide $(17 \mathrm{kDa})$ was detected in serum samples obtained from the patients of both two groups (Fig. 4). Citicoline treatment resulted in a slight decrease of the mean value of the level of this polypeptide in the second group. However, a difference between the mean initial level of this macroglia marker and its post-treatment level did not reach the level of statistical significance $(P=$ $=0.227)$. It means that citicoline treatment exerted nearly no effect on the macrophage function in the ischemic brain, or this effect is rather weak.

It is important to note that basic treatment provided no significant effects on the contents of all above-mentioned studied markers in blood serum of the patients that were members of the control group (Figs. 1-4). Thus, it should be suggested that citicoline may specifically affect certain patterns of stroke-associated pathology during the treatment period.

\section{DISCUSSION}

Current pharmaco-therapeutic strategies for the management of acute ischemic stroke are aimed to target a general pathophysiological cornerstone to prevent secondary complications by restoring the blood flow by thrombolysis or mechanical extraction of blood clots [30]. Anticoagulation via oral administration of the appropriate drugs substantially reduces the risk for ischemic stroke and systemic embolism in patients with AF. However, patients having a stroke, despite being on such therapy with oral anticoagulants, are at a high risk of recurrent ischemic strokes [31]. Thus, alternative or supplementary treatment modalities are needed not only for arterial recanalization and recovery of reperfusion, but also for supporting survival of the entire neurovascular unit and suppression or modulation of the inflammatory response.

Numerous studies have suggested that citicoline is effective in the treatment of CNS disorders, including acute and chronic cerebral ischemia, intracerebral hemorrhage, global cerebral hypoxia, and neurodegenerative pathologies $[12,14,32]$. Citicoline, or cytidine 5'-diphosphocholine (CDPcholine), is a natural endogenous compound and a precursor for the synthesis of phosphatidylcholine, one of the important components of the cell membranes [12, 13]. Citicoline was studied in preclinical and clinical trials and approved in numerous countries for the treatment of acute ischemic stroke as an effective and safe drug [33]. As a multi-target agent, citicoline provides significant neuroprotection by attenuating the glutamate exitotoxicity, oxidative stress, and apoptosis and also helping to preserve the BBB integrity in acute ischemic stroke. In subacute and chronic 
stages of ischemic stroke, citicoline exhibits certain neuroregenerative effects through activation of the processes of neurogenesis and synaptogenesis [32]. In addition, citicoline intensifies angiogenesis, enhances neurotransmitter metabolism, decreases the level of free fatty acids, ameliorates the neurological deficits, restores the learning performance in animals, reduces the glutamate-mediated injury, and stabilizes the phosphatidylcholine level [34]. Several studies suggested that citicoline may enhance the endogenous brain plasticity and intensify the repair phenomena even when it is administered several hours after the ischemic occurrence [34, 35]. Citicoline belongs to the group of nootropics, or "smart" drugs, due to its ability to serve as a cognitive enhancer. The biological activities attributed to citicoline have been shown to play a role in improving the memory abilities, correcting
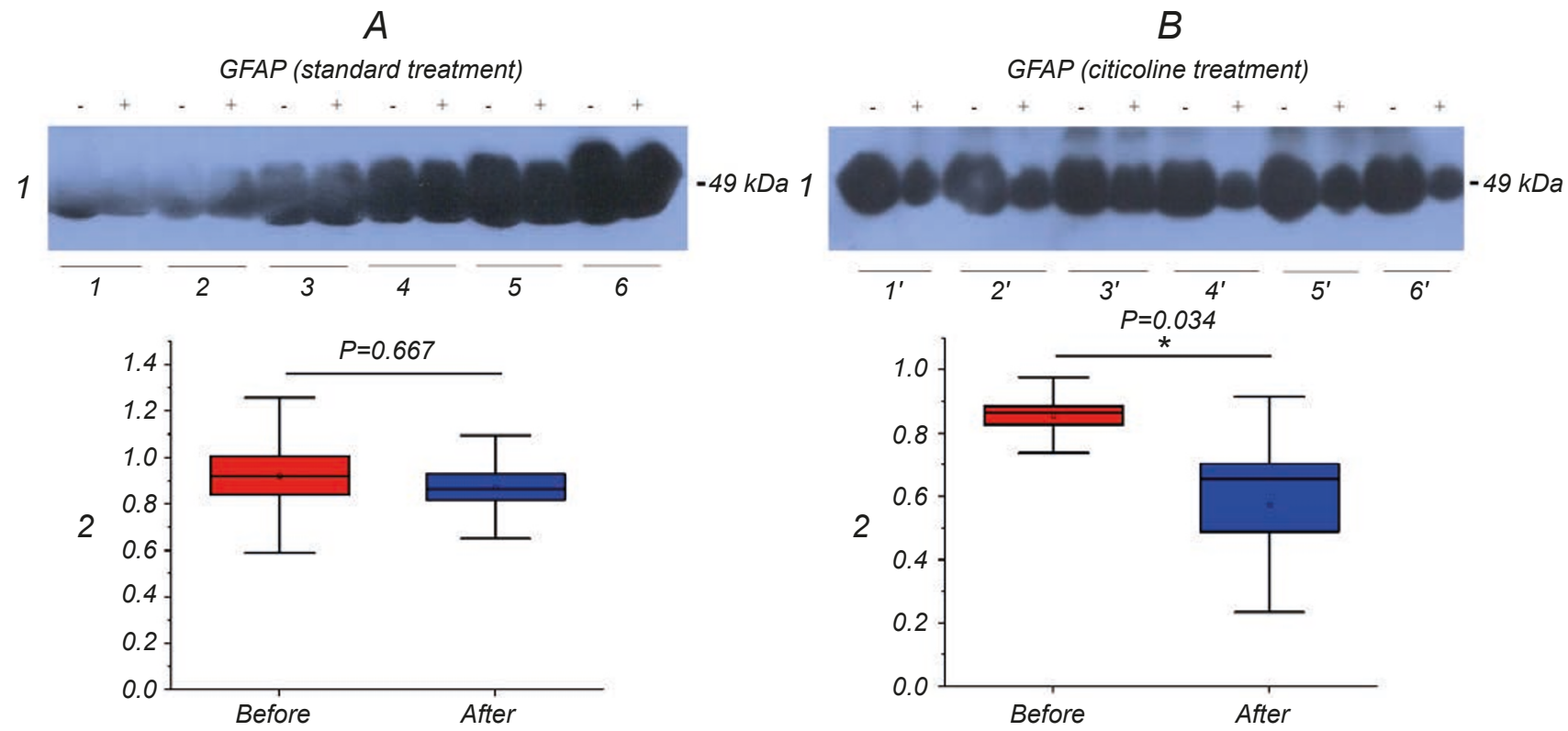

F i g. 1. Immunoblotting of circulating glial fibrillary acidic protein (GFAP) in the blood serum of post-stroke patients with atrial fibrillation that received standard treated (A) and those treated with citicoline. 1) Typical blots of patients of the two groups; 2) results of statistical processing of the respective data; $n=6$ in both groups; *, statistically significant difference $(P<0.05)$.

A

NF-L (standard treatment)
$B$

NF-L (citicoline treatment)

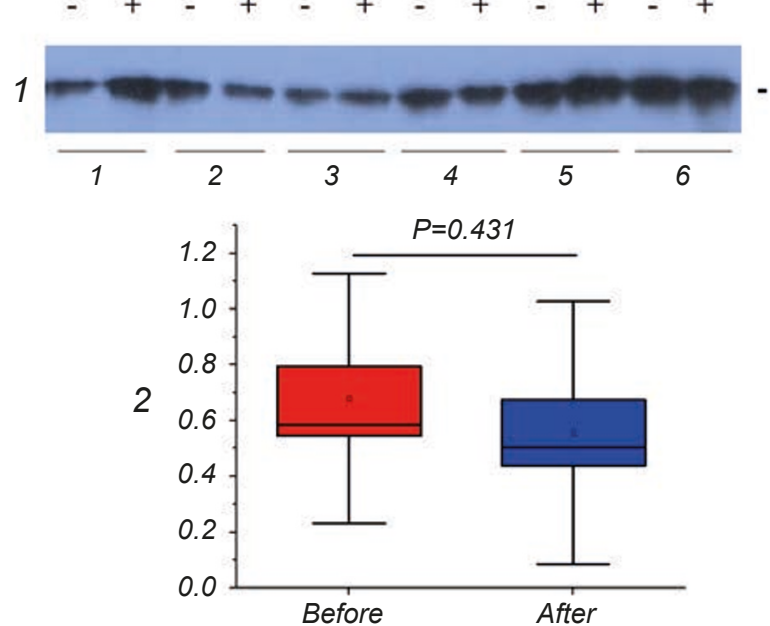

$-68 \mathrm{kDa}$
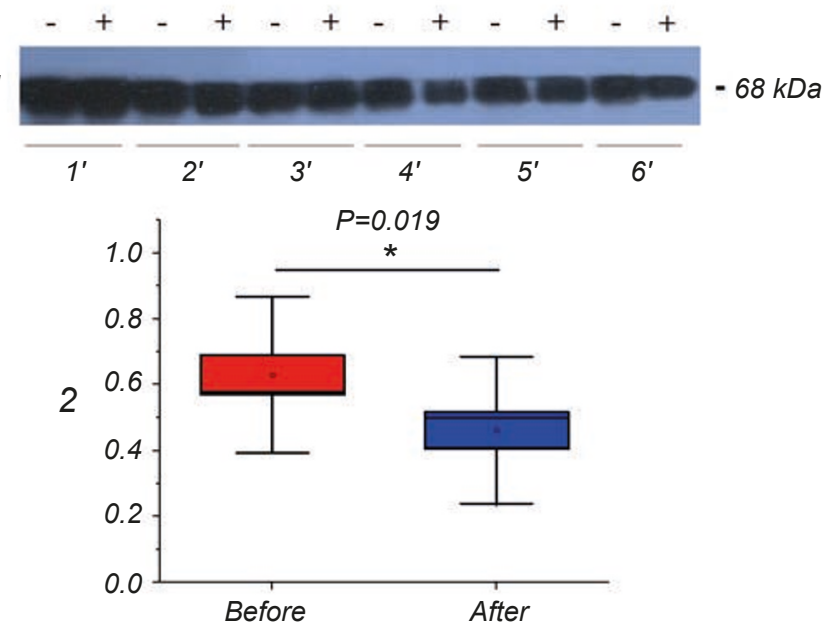

F i g. 2. Immunoblotting of a circulating neurofilament light subunit (NF-L) in the blood serum of patients of the two groups. Designations are similar to those in Fig. 1. 
the learning deficits, and ameliorating cognitive impairments in patients with vascular dementia, Alzheimer's disease, some other cerebrovascular disorders, and elderly-related disorders [36].

It has been well-documented that abrupt deprivation of oxygen and glucose to neuronal tissues due to poor cerebral collateral flow elicits a series of the pathological cascade events leading to intensification of neuronal death. This is associated with poorer outcome after ischemic stroke and larger and faster infarct growth [37]. Moreover, the beat-to-beat variations with reduced cardiac output present in the AF rhythm may result in transient or chronic cerebral hypoperfusion and aggravation of the ischemic conditions [7, 8]. An important effect of citicoline in ischemic stroke provides certain improvement of cerebral perfusion [11]. In our recent study, citicoline was shown to
A

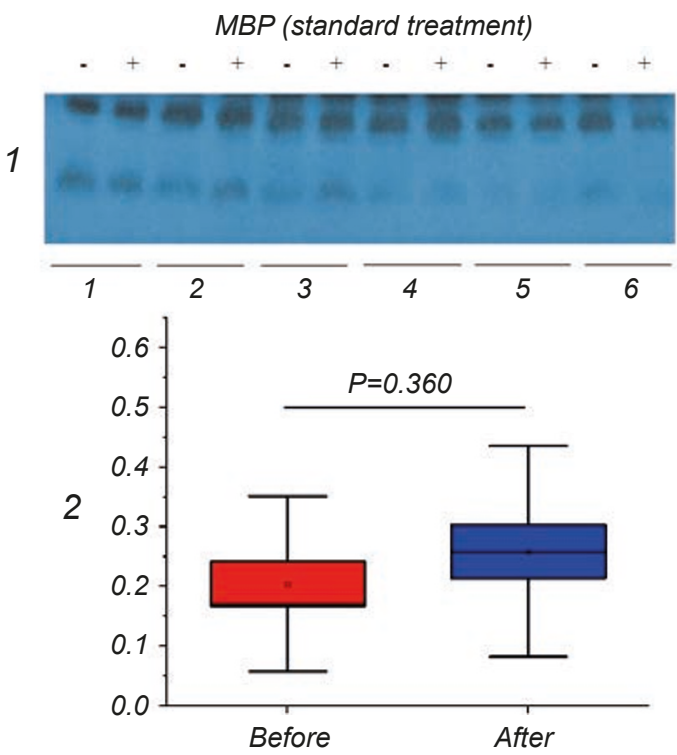

B

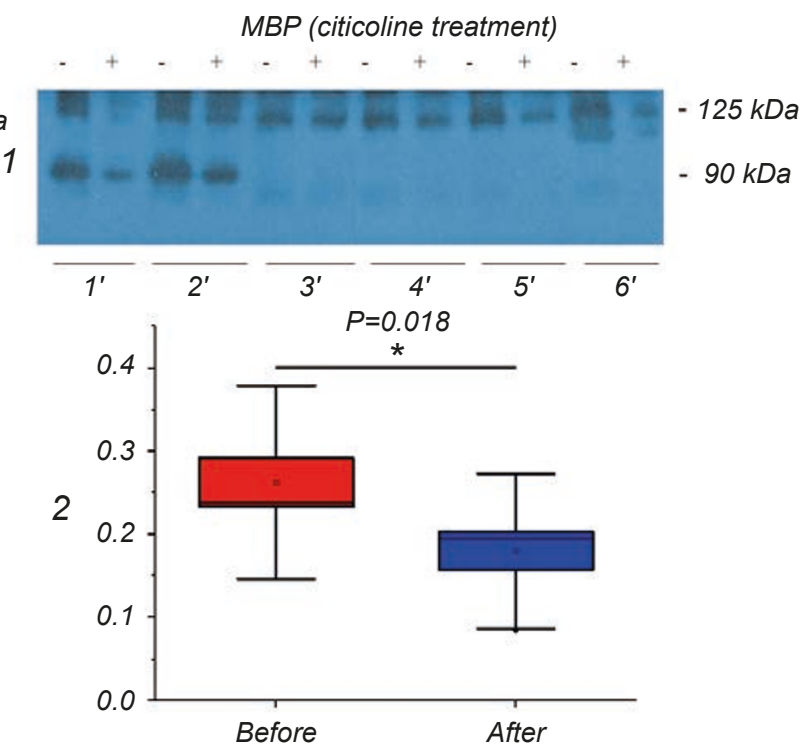

F i g. 3. Immunoblotting of circulating myelin basic protein (MBP) in the blood serum of patients of the two groups. Designations are similar to those in Figs. 1 and 2.

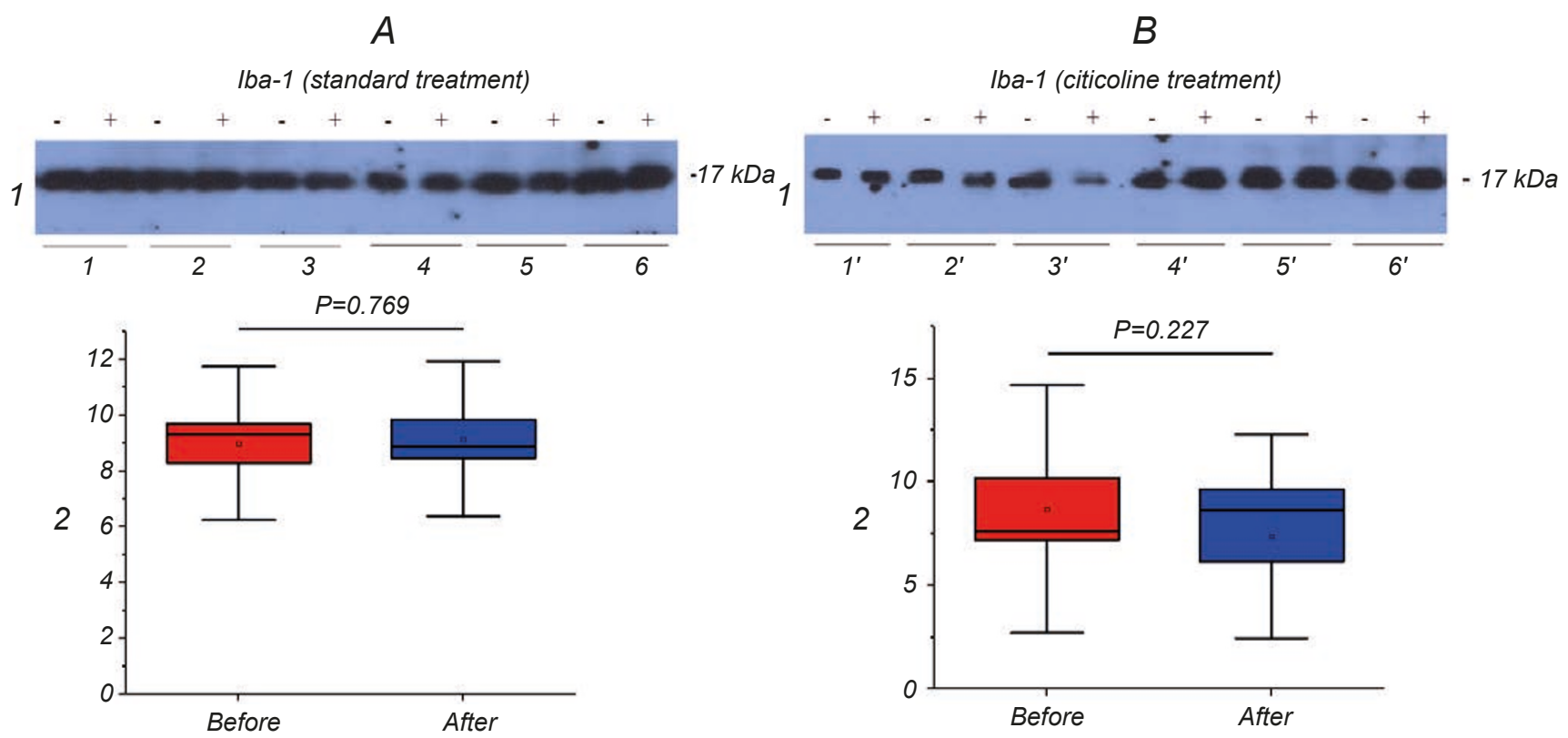

F i g. 4. Immunoblotting of circulating ionized calcium-binding adaptor molecule 1 (Iba-1) in the blood serum of patients of the two groups. Designations are similar to those in Figs. 1-3. 
decrease the circulating levels of angiostatin in patients with ischemic stroke [38]. Angiostatin is a proteolytically-derived fragment of the large protein plasminogen, which acts as a potent inhibitor of migration and proliferation of endothelial cells, and may suppress the collateral formation in ischemic tissues [39]. It was hypothesized that angiostatin-related down-regulation triggers proangiogenic signaling necessary for the initiation of neovascularization and recovery of brain circulation. In addition, citicoline may induce angiogenesis via improving survival of endothelial cells in vascular brain microvessels of humans through the pathways involving ERK1/2 and insulin receptor substrate-1 [40].

Serum biomarkers are promising tools for noninvasive evaluation of the cardiovascular events in patients; many studies have demonstrated correlations between the biomarker levels and clinical outcome or gross pathological findings to inform prognostication of patients with acute ischemic stroke. The inflammatory process associated with stroke events destabilizes the BBB and contributes to a considerable neuroaxonal damage, thereby increasing the release of neuronal, glial and inflammatory markers into the blood [41]. In our study, a panel of neurospecific biomarker levels was assessed in serum of the patients with ischemic strokes and AF. We demonstrated that the tested biomarkers, namely GFAP (a marker of astrocytic injury and BBB disruption), NF-L (that of axonal damage and neuronal degeneration), MBP (that of white matter loss and demyelination), and Iba-1 (a marker of microglia/macrophage activation), can be considered as valuable endpoints of gross pathology that strongly predicts a response to nootropic therapy. These biomarkers were chosen because they may reflect the crosstalk between astrocytes, axons, myelin, and microglia. It is known that astrocytes represent an integral form of neurovascular unit, mediating interactions between the endothelium, neurons, oligodendrocytes, and microglia. However, when astrocytes are activated under stress conditions, normal communication between astrocytes and other cells is interrupted, which results in neuronal loss, death of oligodendrocytes, and subsequent demyelination. These events aggravate inflammation and BBB disruption [42].

Glial fibrillary acidic protein (GFAP) is a brainspecific type-III intermediate filament protein found primarily in mature astrocytes. Various types of brain injuries and neurological disorders are associated with reactive astrogliosis and subsequent GFAP upregulation in the brain. Elevated plasma or serum GFAP levels reflect the astrocytic activation during vasculopathy, cerebral hemorrhage, stroke, and neurodegenerative pathologies (such as Alzheimer's and Parkinson's diseases) [19]. Lafrenaye et al. [25] found that GFAP levels dramatically increased (about 8-fold) in the serum within the initial 6-h-long interval following traumatic brain injury in a pig model. It seems possible that citicoline-based treatment significantly reduced the circulating level of GFAP in post-ischemic patients due to restoration of the astroglial function and recovery of the BBB integrity.

The NF subunits are CNS-enriched cytoskeletal proteins abundantly expressed in long myelinated subcortical white matter axons, where these subunits are essential for the radial growth of axons during the development and for the maintenance of the axon caliber and the velocity of nerve conduction. Neurofilaments belong to the intermediate filament family, comprising a triplet of three subunits, namely NF-L (light, $\sim 68 \mathrm{kDa}$ ), NF-M (medium, $\sim 145 \mathrm{kDa}$ ), and NF-H (heavy, $\sim 200 \mathrm{kDa}$ ). However, among the three NF subunits, NF-L is the most abundant and essential component of the neurofilament core, functioning as a backbone to which NF-M and NF-H are connected [43]. Blood levels of NFs were shown to be biomarkers that monitor and predict the progression of various neurological diseases $[17,18]$. The NF-L levels are general indicators of neuroaxonal damage. Elevated levels of NF-L are detectable in the serum and were described in a number of CNS diseases, like multiple sclerosis, dementia, stroke, traumatic brain injury, chronic inflammatory demyelination neuropathy, cerebral small vessel disease, etc. [41]. Ischemic stroke has recently been shown to be accompanied by persistent elevations of the serum NF-L level, which correlated with the infarct volumes and recurrent ischemic lesions [44]. In our study, we observed that citicoline treatment noticeably reduced the serum NF-L levels in patients with ischemic stroke and AF. Therefore, similarly to GFAP, the amount of this subunit holds great promise as a biomarker for monitoring the responses on nootropic therapy. It is important to note that GFAP and NF-L levels in the blood were recently utilized for assessing neurological manifestations of COVID-19 [45]. Elevated plasma GFAP levels correlated with the intensity of vasculopathy and 
cerebral hemorrhage extent in COVID-19 and may demonstrate a potential utility in identifying patients who should be closely monitored for neurological complications, such as hemorrhage and stroke. The blood NF-L concentration was higher in patients with COVID-19 between days 1 and 7, which might reflect neurological dysfunction or neurodegeneration and COVID-19-associated delirium resulted from direct related to SARS-CoV2-related neuronal involvement. In an earlier study of CDP-choline-assisted treatment of hypertensive intracerebral hemorrhage, citicoline has been shown to reduce the death rate of neurons via suppressing generation of soluble phosphatidyl choline and effectively weakening the activity of phosphatidase A2. This agent inhibited inflammatory factors and improved the prognosis for the patients [46]. Moreover, citicoline inhibited the release of GFAP, whose level positively correlated with the volume of brain edema in patients with cerebral hemorrhage. Thus, these observations are consistent with our findings, indicating that the application of citicoline can significantly down-regulate the serum GFAP content.

Oligodendrocyte cell death and myelin disruption are typical histopathological features of the ischemic brain lesion, which begin as early as $30 \mathrm{~min}$ after the stroke and further progress up to the formation of a glial scar or tissue necrosis [47]. Thus, information about the state of myelin in stroke may play an important role for treatment monitoring and predicting rehabilitation outcomes. Oligodendrocytes are highly sensitive to various stress-related factors, including the intensity of oxidative stress, excitotoxicity, axonal injury, and inflammation. All these factors induce oligodendrocyte cell death and contribute to the post-stroke demyelination process [20]. While the white matter plays a highly essential role in signal transmission and communication between different brain regions, oligodendrocyte cell death and axonal demyelination may result in severe neurological dysfunction, including motor disorders, impaired cognitive abilities, mental disorders, and neurodegeneration [48].

MBP is known to be the second most abundant protein in the CNS (after the proteolipid protein); it constitutes about $30 \%$ of the protein component in the CNS. The destroyed myelin is engulfed by macrophages as a vesicular material. However, elevated levels of MBP in the serum were observed in traumatic brain injury, multiple sclerosis, stroke, and a variety of other neurological conditions [21]. It was evidenced that MBP fragments released during myelin breakdown undergo enzymatic modification in the blood and, additionally, in the kidneys [20]. Thus, protection of oligodendrocytes and activation of remyelination are indispensable for restorative therapeutic strategy and neurobehavioral function recovery after ischemic stroke. One of the key findings in our study was the ability of CDPcholine to inhibit MBP release into the circulation in post-stroke patients. However, this result of our study probably indicates that reduced MBP serum levels caused by citicoline-assisted treatment during the examined post-stroke period are rather strongly related to the glioprotective effects of nootropics therapy and partial recovery of the BBB integrity. Skripuletz et al. [49] highlighted the mechanisms of stimulation of the brain phospholipid and myelin synthesis by citicoline. It was hypothesized that, during remyelination, oligodendrocyte precursor cells proliferate, migrate into the demyelinated areas, and finally differentiate into myelinating oligodendrocytes. In the presence of CDP-choline, proliferation of these precursor cells is intensified; thus, more and more these sells can migrate into the demyelinated area, and more axons are remyelinated. Meanwhile, Grieb [50] described that citicoline ameliorates the disease course and exerts beneficial effects on myelin, oligodendrocytes, and axons in a model of experimental autoimmune damage. Thus, our results confirm the efficacy of citicoline-based therapeutic strategy to target demyelination, which is important for improving long-term neurological functional recovery in stroke patients.

Following acute ischemic stroke, neuroinflammation develops, which may exacerbate further injury resulting in cell death. At the same time, neuroinflammation may play a beneficial role by promoting recovery [5]. Microglia comprises resident immune cells of the CNS, which play a central role in the initiation and propagation of the inflammatory responses. Activated microglial units act similarly to macrophages during systemic inflammation and demonstrate the ability to phagocytose deleterious cellular debris. On the other hand, microglial cells may exert cytotoxic effects by releasing excesive amounts of reactive oxygen and nitric species, inflammatory cytokines, and proteolytic enzymes, which trigger a considerable neuronal damage [22]. Activated microglia upregulates cell surface markers, which facilitates migration of the latter to the injury site. Little is 
known, however, about how pathophysiological manifestations of stroke correlate with alterations of the circulating levels of microglia biomarkers, and is there an association of these levels with the severity of ischemic tissue damage. In our study, we measured serum levels of the ionized calcium-binding adaptor molecule-1 (Iba-1) as a novel marker of microglia/macrophage activation in the ischemic brain [25]. To our knowledge, this is the first study where the circulating Iba-1 level has been used to monitor microglial activation during citicoline-based treatment of the patients with ischemic stroke. In contrast to other studied neuronal and glial markers, we, however, showed that citicoline administration exerted no significant effect on the blood levels of Iba-1. This may indicate that citicoline preserves an important functional significance of microglia, which is needed to resolve local inflammation, to clear cellular debris, and to provide protective factors reducing cell injury in the ischemic brain.

Collectively, we obtained notable observations indicating clear relationships between citicoline treatment and changes in the levels of neurological biomarkers, which are released from the brain (from glia, myelin, and neurons) in the blood after a stroke event. Nonetheless, it is still necessary to translate these changes into clinical consideration. Though a number of the candidates for reliable clinical indices in ischemic stroke have been identified among blood circulating biomarkers, so far none of those is widely used in the clinical practice. We believe that, with a further well-designed study and careful validation, successful identification of blood biomarkers, supporting the clinical diagnosis of stroke will help to estimate the risk of disease in patients, guide the treatment course, improve the care, and increase the efficiency of prognosis.

The study was performed within the framework of the Agreement on scientific collaboration between the Palladin Institute of Biochemistry of NAS of Ukraine (Kyiv, Ukraine) and the Dnipropetrovsk Medical Academy, Ministry of Public Health of Ukraine (Dnipro, Ukraine) (2019-2021).

Written informed consent was obtained from all patients to participate in the study. All study procedures and protocols used in the investigation were reviewed and approved by the Ethical Committee of the Dnipropetrovsk Medical Academy, Ministry of Public Health of Ukraine (protocol No. 6, 07/02/2018).
The authors, O. V. Kuryata, Yu. S. Kushnir, V. S. Nedzvetsky, V. V. Korsa, and A. A. Tykhomyrov, confirm the absence of any conflicts over commercial or financial relations, relations with organizations or individuals that could in any way be related to the study, and also in interrelations between the co-authors.

\section{REFERENCES}

1. J. Kornej, C. S. Börschel, E. J. Benjamin, and R. B. Schnabel, "Epidemiology of atrial fibrillation in the $21 \mathrm{st}$ century: novel methods and new insights," Circ. Res., 127, No. 1, 4-20 (2020); doi:10.1161/CIRCRESAHA.120.316340.

2. A. M. Alshehri, "Stroke in atrial fibrillation: review of risk stratification and preventive therapy," J. Family Community Med., 26, No. 2, 92-97 (2019); doi:10.4103/ jfcm.JFCM_99_18.

3. D. A. Dulli, H. Stanko, and R. L. Levine, "Atrial fibrillation is associated with severe acute ischemic stroke," Neuroepidemiology, 22, No. 2, 118-123 (2003); doi: 10.1159/000068743.

4. E. S. Donkor, "Stroke in the 21 st century: a snapshot of the burden, epidemiology, and quality of life," Stroke Res. Treat., 2018, 3238165, (2018); doi:10.1155/ 2018/3238165.

5. R. L. Jayaraj, S. Azimullah, R. Beiram, et al., "Neuroinflammation: friend and foe for ischemic stroke," $J$. Neuroinflammation, 16, No. 1, 142 (2019); doi: 10.1186/ s12974-019-1516-2.

6. H. Stefansdottir, D. O. Arnar, T. Aspelund, et al., "Atrial fibrillation is associated with reduced brain volume and cognitive function independent of cerebral infarcts," Stroke, 44, No. 4, 1020-1025 (2013); doi:10.1161/ STROKEAHA.12.679381.

7. A. Saglietto, M. Matta, F. Gaita, et al., "Stroke-independent contribution of atrial fibrillation to dementia, a meta-analysis," Open Heart, 6, No. 1, e000984 (2019); doi: 10.1136/openhrt-2018-000984.

8. M. Gardarsdottir, S. Sigurdsson, T. Aspelund, et al., "Atrial fibrillation is associated with decreased total cerebral blood flow and brain perfusion," Europace, 20, No. 8, 1252-1258 (2018); doi:10.1093/europace/eux220.

9. R. N. Kalaria, "Risk factors and neurodegenerative mechanisms in stroke related dementia," Panminerva Med., 54, No. 3, 139-148 (2012).

10. T. A. Manolis, A. A. Manolis, E. J. Apostolopoulos, et al., "Atrial fibrillation and cognitive impairment: an associated burden or burden by association?," Angiology, 71, No. 6, 498-519 (2020); doi:10.1177/ 0003319720910669.

11. R. M. Ferreira Lisboa Da Silva, C. Madeira Miranda, T. Liu, et al., "Atrial fibrillation and risk of dementia: epidemiology, mechanisms, and effect of anticoagulation," Front. Neurosci., 13, Article 18, 1-7 (2019); doi: 10.3389/fnins.2019.00018. 
12. P. Jasielski, F. Piędel, M. Piwek, et al., "Application of citicoline in neurological disorders: a systematic review," Nutrients, 12, No. 10, 3113 (2020); doi: 10.3390/ nu12103113.

13. L. F. Borkenhagen and E. P. Kennedy, "The enzymatic synthesis of cytidine diphosphate choline," J. Biol. Chem., 227, No. 2, 951-962 (1957).

14. J. J. Secades, J. Alvarez-Sabín, J. Castillo, et al., "Citicoline for acute ischemic stroke: a systematic review and formal meta-analysis of randomized, double-blind, and placebo-controlled trials," J. Stroke Cerebrovasc Dis., 25, No. 8, 1984-1996 (2016); doi:10.1016/j.jstrokecere brovasdis.2016.04.010.

15. O. Y. Glushakova, A. V. Glushakov, E. R. Miller, et al., "Biomarkers for acute diagnosis and management of stroke in neurointensive care units," Brain Circ., 2, No. 1, 28-47 (2016); doi:10.4103/2394-8108.178546.

16. G. C. Jickling and F. R. Sharp, "Blood biomarkers of ischemic stroke," Neurotherapeutics, 8, No. 3, 349-360 (2011); doi: 10.1007/s13311-011-0050-4.

17. M. Khalil, C. E. Teunissen, M. Otto, et al., "Neurofilaments as biomarkers in neurological disorders," Nat. Rev. Neurol., 14, No. 10, 577-589 (2018); doi: 10.1038/ s41582-018-0058-z.

18. Y. Lee, B. H. Lee, W. Yip, et al., "Neurofilament proteins as prognostic biomarkers in neurological disorders," Curr. Pharm. Des., 25, No. 43, 4560-4569 (2020); doi: $10.2174 / 1381612825666191210154535$.

19. Z. Yang and K. K. Wang, "Glial fibrillary acidic protein: from intermediate filament assembly and gliosis to neurobiomarker," Trends Neurosci., 38, No. 6, 364-374 (2015); doi:10.1016/j.tins.2015.04.003

20. Y. Wang, G. Liu, D. Hong, et al., "White matter injury in ischemic stroke," Prog. Neurobiol., 141, 45-60 (2016); doi:10.1016/j.pneurobio.2016.04.005.

21. N. Wąsik, B. Sokół, M. Hołysz, et al., "Serum myelin basic protein as a marker of brain injury in aneurysmal subarachnoid haemorrhage," Acta Neurochir. (Wien), 162, No. 3, 545-552 (2020); doi: 10.1007/s00701-01904185-9.

22. J. Lee, G. Hamanaka, E. H. Lo, et al., "Heterogeneity of microglia and their differential roles in white matter pathology," CNS Neurosci. Ther., 25, No. 12, 1290-1298 (2019); doi:10.1111/cns.13266.

23. S. Xu, J. Lu, A. Shao, et al., "Glial cells: role of the immune response in ischemic stroke," Front. Immunol., 11, 294 (2020); doi:10.3389/fimmu.2020.00294.

24. Y. Sasaki, K. Ohsawa, H. Kanazawa, et al., "Ibal is an actin-cross-linking protein in macrophages/microglia," Biochem. Biophys. Res. Commun., 286, No. 2, 292-297 (2001); doi:10.1006/bbrc.2001.5388.

25. A. D. Lafrenaye, S. Mondello, K. K. Wang, et al., "Circulating GFAP and Iba-1 levels are associated with pathophysiological sequelae in the thalamus in a pig model of mild TBI," Sci. Rep., 10, No. 1, 13369 (2020); doi:10.1038/s41598-020-70266-w.

26. "Corrigendum to: 2020 ESC Guidelines for the diagnosis and management of atrial fibrillation developed in collaboration with the European Association of CardioThoracic Surgery (EACTS)," Eur. Heart J., 42, No. 5, 507 (2020); doi: 10.1093/eurheartj/ehaa798.
27. G. Montalescot, U. Sechtem, S. Achenbach, et al., "2013 ESC guidelines on the management of stable coronary artery disease: The Task Force on the management of stable coronary artery disease of the European Society of Cardiology," Eur. Heart J., 34, No. 38, 2949-3003 (2013). doi: 10.1093/eurheartj/eht296.

28. W. J. Powers, A. A. Rabinstein, T. Ackerson, et al., "Guidelines for the early management of patients with acute ischemic stroke: 2019 Update to the 2018 Guidelines for the early management of acute ischemic stroke: A Guideline for healthcare professionals from the American Heart Association/American Stroke Association," Stroke, 50, No. 12, e344-e418 (2019); doi. org/10.1161/STR.0000000000000211.

29. C. M. Stoscheck, "Quantitation of protein," Methods Enzymol., 182, 50-68 (1990); doi: 10.1016/0076-6879 (90)82008-p.

30. X. Yi, J. Lin, Z. Han, et al., "Preceding antithrombotic treatment is associated with acute ischemic stroke severity and functional outcome at 90 days among patients with atrial fibrillation," J. Stroke. Cerebrovasc. Dis., 28, No. 7, 2003-2010 (2019); doi:10.1016/j.jstrok ecerebrovasdis.2019.03.028.

31. Y. D. Cheng, L. Al-Khoury, and J. A. Zivin, "Neuroprotection for ischemic stroke: two decades of success and failure," NeuroRx., 1, No. 1, 36-45 (2004); doi:10.1602/neurorx.1.1.36.

32. M. Y. Martynov and E. I. Gusev, "Current knowledge on the neuroprotective and neuroregenerative properties of citicoline in acute ischemic stroke," J. Exp. Pharmacol., 7, 17-28 (2015); doi:10.2147/JEP.S63544.

33. J. J. Secades, "Citicoline: pharmacological and clinical review, 2016 update," Rev. Neurol., 63, No. S03, S1-S73 (2016).

34. W. M. Clark, "Efficacy of citicoline as an acute stroke treatment," Expert Opin. Pharmacother., 10, No. 5, 839846 (2009); doi:10.1517/17460440902835475.

35. K. Overgaard, "The effects of citicoline on acute ischemic stroke: a review," J. Stroke. Cerebrovasc. Dis., 23, No. 7, 1764-1769 (2014); doi:10.1016/j.jstrokecere brovasdis.2014.01.020.

36. J. Alvarez-Sabín and G. C. Román, "Citicoline in vascular cognitive impairment and vascular dementia after stroke," Stroke, 42, No. 1, 40-43 (2011); doi:10.1161/ STROKEAHA.110.606509.

37. P. Deb, S. Sharma and K. M Hassan, "Pathophysiologic mechanisms of acute ischemic stroke: an overview with emphasis on therapeutic significance beyond thrombolysis," Pathophysiology, 17, No. 3, 197-218 (2010); doi:10.1016/j.pathophys.2009.12.001.

38. A. A. Tykhomyrov, Y. S. Kushnir, V. S. Nedzvetsky, et al., "Citicoline affects serum angiostatin and neurospecific protein levels in patients with atrial fibrillation and ischemic stroke," Ukr. Biochem. J., 91, No. 5, 34-45 (2019); doi: https://doi.org/10.15407/ubj91.05.034.

39. T. Matsunaga, D. W. Weihrauch, M. C. Moniz, et al., "Angiostatin inhibits coronary angiogenesis during impaired production of nitric oxide," Circulation, 105, No. 18, 2185-2191 (2002); doi:10.1161/01.cir. 0000015856.84385.e9. 
40. J. Krupinski, M. Abudawood, S. Matou-Nasri, et al., "Citicoline induces angiogenesis improving survival of vascular/human brain microvessel endothelial cells through pathways involving ERK1/2 and insulin receptor substrate-1,"Vasc. Cell, 4, No. 1, 20 (2012); doi: 10.1186/2045-824X-4-20.

41. H. H. Nielsen, S. B. Soares, S. S. Høgedal, et al., "Acute neurofilament light chain plasma levels correlate with stroke severity and clinical outcome in ischemic stroke patients," Front. Neurol., 11, 448 (2020); doi:10.3389/ fneur.2020.00448.

42. S. Köhler, U. Winkler, and J. Hirrlinger, "Heterogeneity of astrocytes in grey and white matter," Neurochem. Res., 46, No. 1, 3-14 (2021); doi: 10.1007/s11064-01902926-X.

43. A. Yuan, M. V. Rao, Veeranna, and R. A. Nixon, "Neurofilaments and neurofilament proteins in health and disease," Cold Spring Harb. Perspect. Biol., 9, No. 4, a018309 (2017); doi:10.1101/cshperspect.a018309.

44. S. Tiedt, M. Duering, C. Barro, et al., "Serum neurofilament light: a biomarker of neuroaxonal injury after ischemic stroke," Neurology, 91, No. 14, e1338-e1347 (2018); doi:10.1212/WNL.0000000000006282.

45. J. Cooper, S. Stukas, P. L. Hoiland, et al., "Quantification of neurological blood-based biomarkers in critically ill patients with coronavirus disease 2019," Crit. Care. Explor., 2, No. 10, e0238 (2020); doi:10.1097/ CCE.0000000000000238.

46. Y. Zang, A. Liu, and L. Qi "The cytological mechanism and effects of hypertensive cerebral hemorrhage treatment by citicoline on serum GFAP and copeptin level," Eur. J. Inflam., 17, 1-8 (2019); https://doi. org/10.1177/2058739219867244.

47. M. Y. Khodanovich, A. A. Kisel, A. E. Akulov, et al., "Quantitative assessment of demyelination in ischemic stroke in vivo using macromolecular proton fraction mapping," J. Cereb. Blood Flow. Metab., 38, No. 5, 919-931 (2018); doi:10.1177/0271678X18755203.

48. H. Sh, X. Hu, R. K. Leak, et al., "Demyelination as a rational therapeutic target for ischemic or traumatic brain injury," Exp. Neurol., 272, 17-25 (2015); doi:10. 1016/j.expneurol.2015.03.017.

49. T. Skripuletz, R. A. Linker, and M. Stangel, "The choline pathway as a strategy to promote central nervous system (CNS) remyelination," Neural. Regen. Res., 10, No. 9, 1369-1370 (2015); doi:10.4103/1673-5374.165498.

50. P. Grieb, "Beneficial effects of exogenous CDP-choline (citicoline) in EAE," Brain, 138, No. 11, e388 (2015); doi:10.1093/brain/awv140. 